\title{
Transfer of Bone-Marrow-Derived Mesenchymal Stem Cells Influences Vascular Remodeling and Calcification after Balloon Injury in Hyperlipidemic Rats
}

\author{
Jianquan Liao, ${ }^{1}$ Xiaochun Chen, ${ }^{2}$ Yongdong Li, ${ }^{2}$ Zhiping Ge, ${ }^{2}$ Hongyu Duan, ${ }^{2}$ \\ Yunzeng Zou, ${ }^{1}$ and Junbo $\mathrm{Ge}^{1}$ \\ ${ }^{1}$ Shanghai Institute of Cardiovascular Diseases, Zhongshan Hospital, Fudan University, Shanghai 200032, China \\ ${ }^{2}$ Department of Cardiology, The Third Affiliated Hospital, Inner Mongolia Medical College, BaoTou, Inner Mongolia 014010, China \\ Correspondence should be addressed to Xiaochun Chen, chen1104003@yahoo.com.cn \\ and Junbo Ge, ge.junbo2@zs-hospital.sh.cn
}

Received 19 December 2011; Revised 27 February 2012; Accepted 29 February 2012

Academic Editor: Monica Fedele

Copyright () 2012 Jianquan Liao et al. This is an open access article distributed under the Creative Commons Attribution License, which permits unrestricted use, distribution, and reproduction in any medium, provided the original work is properly cited.

Bone-marrow-derived mesenchymal stem cells (BM-MSCs) were found to markedly increase atherosclerotic lesion size. The aim of this paper was to investigate whether BM-MSCs contribute to vascular remodeling and calcification after balloon injury in hyperlipidemic rats. Labeled BM-MSCs were found in the lesion of hyperlipidemic rats after balloon injury. Comparing injury group, transferred BM-MSCs significantly triggered vascular negative remodeling, characterized by the changes of remodeling index $(0.628 \pm 0.0293$ versus $0.544 \pm 0.0217)$, neointimal area $\left(0.078 \pm 0.015 \mathrm{~mm}^{2}\right.$ versus $\left.0.098 \pm 0.019 \mathrm{~mm}^{2}\right)$, PCNA index $(23.91 \pm$ $6.59 \%$ versus $43.11 \pm 5.31 \%$ ), and percentage of stenosis $(18.20 \pm 1.09 \%$ versus $30.58 \pm 1.21 \%)$. Apparent vascular calcification was detected in medial layers at 6 weeks after balloon angioplasty, which may be associated with upregulation of bone morphogenetic protein-2 (BMP-2). Our data indicated that unselected BM-MSCs transfer may induce vascular remodeling and calcification after balloon injury in hyperlipidemic rats.

\section{Introduction}

Cellular therapy has been an area of intense research over few decades, which is widely used for treatment of cardiovascular disease. Bone-marrow-derived mesenchymal stem cells (BMMSCs) were isolated from adult bone marrow, along with the ability for self-renewal and multilineage differentiation into cardiomyocytes, smooth muscle cells, endothelium, osteoblasts, and chondroblasts $[1,2]$. Accumulating evidence from a growing body of animal studies demonstrated the extensive capacity of MSCs to engraft, differentiate and produce substantial functional recovery [3]. Transplantation has been considered as a potential clinical strategy for the treatment of ischemic heart diseases and heart failure.

Although a growing body of evidence from animal experiments and clinical trials indicated the beneficial therapeutic potential of MSCs in cardiovascular diseases, the safety of MSCs transplantation is a critical problem that should be kept in mind. The findings that MSCs transplantation could result in tumor formation and alteration of electrophysiologic properties caused wide concerns $[4,5]$. Furthermore, unexpected severe intramyocardial calcification was elicited by direct transplantation of unselected bone marrow cells into acutely infarcted myocardium [6]. It has been demonstrated that transfer of bone marrow and endothelial progenitor cells accelerated atherosclerosis and influenced the plaque stability [7]. Increasing evidence indicates a link between MSCs therapy and pathogenesis of atherosclerosis $[8,9]$.

Our previous studies demonstrated that oxidized LDL synergistically promoted osteodifferentiation of bone-marrow-derived MSCs in response to osteogenic inductor. In this study, we investigated the effects of transfer of unselected BM-MSCs on vascular remodeling and calcification in the hyperlipidemic rat. 


\section{Method}

2.1. Preparation of BM-MSCs. Unselected BM-MSCs were extracted from male Sprague-Dawley (SD) rats (150 200 g) femurs by flushing the bone marrow cavities with Dulbecco's modified Eagle's medium-low glucose (DMEM) and cultured in DMEM supplemented with 10\% fetal bovine serum (FBS). After $24 \mathrm{~h}$, the medium was discarded to remove hematopoietic stem cells and nonadherent cells. Medium was changed every $1 \sim 2$ days. Cells became almost confluent after 8 days and were trypsinized with $0.25 \%$ trypsin containing $1 \mathrm{mM}$ EDTA for $3 \mathrm{~min}$ at $37^{\circ} \mathrm{C}$. All experiments were performed on cells cultured for up to $3 \sim 5$ passages. BM-MSCs were identified as CD90-positive and CD34-negative by flow cytometry.

2.2. Animals. All animal experiments were approved by the Animal Care and Use Committee of Fudan University according to the Guide for the Care and Use of Laboratory Animals, published by the US National Institutes of Health (NIH publication no. 85-23, revised 1996). Animals were fed with high cholesterol diet containing $10 \%$ fat, $5 \%$ cholesterol, $0.5 \%$ sodium cholate, and $0.2 \%$ propylthiouracil and received vitamin $\mathrm{D}_{3}$ (600,000 U/kg body weight) for 1 week before balloon denudation. Female SD rats were randomly allocated to three groups: (1) rats without denudation were fed with high cholesterol diet (Control, $n=8$ ); (2) rats were subjected to balloon denudation and fed with high cholesterol diet (Injury group, $n=8$ ); (3) rats were subjected to balloon denudation and BM-MSCs transplantation plus with high cholesterol diet (MSC group, $n=8$ ). Rats were anesthetized with ketamine $(150 \mathrm{mg} / \mathrm{kg}$ body weight IP) and heparinized with $100 \mathrm{U} / \mathrm{kg}$ heparin sodium before procedures. A well-established rat model of aortic artery balloon injury was used in this study. The abdominal aorta was isolated and stripped of adventitia. A $6 \mathrm{~F}$ balloon catheter was introduced through incision under the renal artery bifurcation about $1 \mathrm{~cm}$ and advanced into the thoracic aorta. The balloon was inflated with saline and pulled back to the abdominal aorta. After balloon denudation was performed for 3 times, saline or BMSCs labeled by DAPI were injected before removal of the catheter. After balloon denudation, rats were fed with high cholesterol diet containing $10 \%$ fat, $5 \%$ cholesterol, $0.5 \%$ sodium cholate, and $0.2 \%$ propylthiouracil for 6 weeks after balloon denudation. In addition, rats without denudation were fed with a normal diet to compare with those with a high cholesterol diet.

2.3. Analyses of Plasma Lipid. After feeding, the rats were fasted overnight and the samples were collected and centrifuged at $3000 \mathrm{rpm}$ for $15 \mathrm{~min}$. The plasma lipid profile was determined by measuring the contents of total cholesterol (TC) and low-density lipoprotein (LDL) by standard enzymatic procedures using reagent kits.

2.4. Histology. Paraffin sections of vascular tissue were stained with hematoxylin-eosin for morphometric analysis as previously described [10]. Histomorphometric parameters, including luminal area (LA), internal elastic lamina area (IELA), external elastic lamina area (EELA), and neointimal area were defined and determined using the software of Leica QWin Plus as previously described [11]. The chronic variation of the area surrounded by the external elastic lamina was evaluated by remodeling index compared with a reference normal segment upstream from the lesion site, which was calculated as a percentage as follows: EELA(lesion)/EELA (reference). The percentage of stenosis was calculated as $($ IELA-LA/IELA) $\times 100$.

2.5. Von Kossa Stain. Von Kossa stain (Sigma-Aldrich, USA) was performed to evaluate abnormal deposits of calcium within vascular wall. Paraffin-fixed tissue sections were incubated with silver nitrate solution under a strong light. The calcium was replaced with silver deposit, visualized as metallic silver. Procedural details are described elsewhere [6].

2.6. Immunohistochemistry. Proliferative activity was determined in vascular tissue using analysis of proliferating cell nuclear antigen (PCNA) expression defined immunohistochemically. In brief, tissue sections were quenched for endogenous peroxidase and antigen-retrieved in $0.01 \mathrm{M}$ citrate buffer ( $\mathrm{pH}$ 6.0) for $15 \mathrm{~min}$ in a microwave oven at $100^{\circ} \mathrm{C}$. The sections were blocked with normal goat serum and then incubated with mouse anti-PCNA antibody (Boster Company, China) overnight in a humid chamber at $4^{\circ} \mathrm{C}$. After incubation with biotinylated goat anti-mouse IgG, sections were visualized by using avidin-biotin horseradish peroxidase visualization system. Sections were then counterstained with haematoxylin. Images were recorded using Leica Qwin Plus.

2.7. Immunofluorescence Staining. Immunofluorescence staining was performed according to standard procedures. Briefly, vascular tissue sections were deparaffinized and hydrated. After preincubation with normal goat serum, sections were incubated with rabbit anti-BMP-2 antibody (Boster Company, China) overnight in humid chamber at $4^{\circ} \mathrm{C}$ followed by incubation with appropriate FITC-linked secondary antibody. Images were captured by using Leica Qwin Plus.

2.8. Statistical Analysis. Data were presented as means \pm SEM. Comparison was performed using one-way analysis of variance (ANOVA) with Tukey test for post hoc analysis to determine the difference among groups. $P<0.05$ was considered statistically significant.

\section{Result}

3.1. Identification of BM-MSCs Prepared for Transplantation. For transplantation, unselected BM-MSCs were isolated from bone marrow according to standard technique. A few small, bright, round cells were present in the primary culture, which gradually disappeared by the second generation or later (Figure 1(a)). BM-MSCs were characterized as 


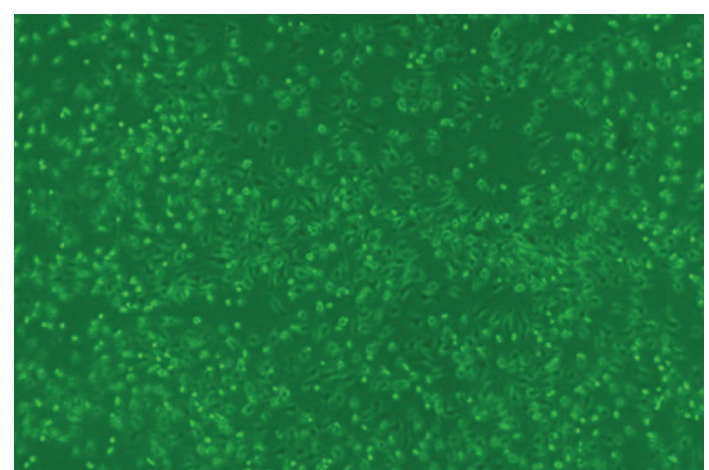

(a)

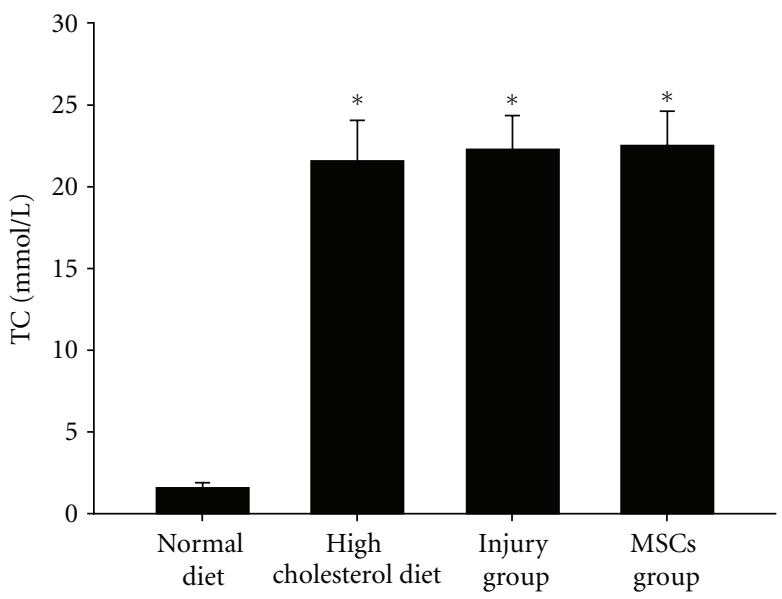

(c)

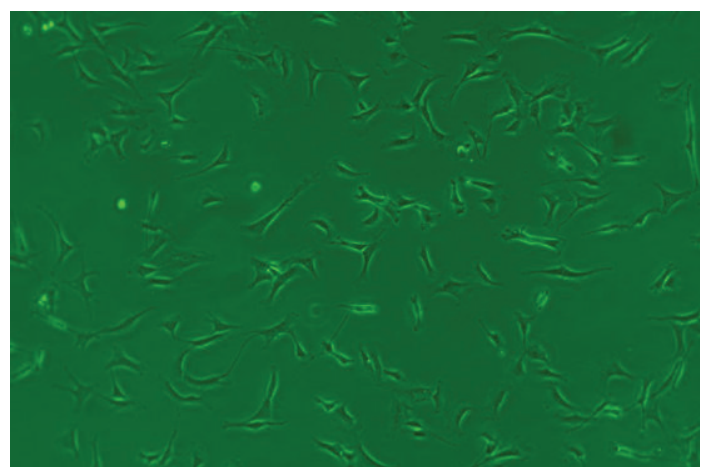

(b)

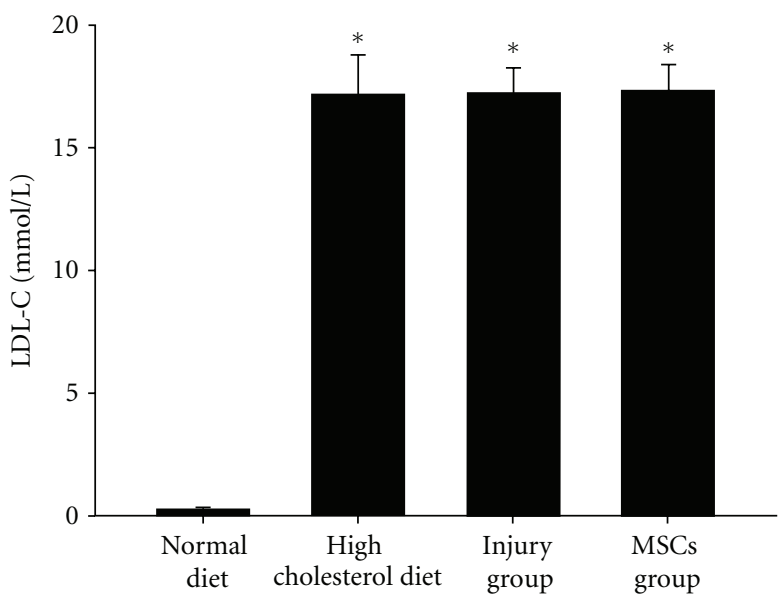

(d)

FIGURE 1: Identification of BM-MSCs and the profile of lipid level in cholesterol-rich fed rats. Phase contrast microscopy depicted that a few small, bright, round cells were present in the primary culture (a). Phase contrast microscopy revealed that BM-MSCs were characterized as a fibroblast-like spindle-shaped morphology (b). Baseline of serum lipid content before received cholesterol-rich diet did not significantly differ among three groups. Cholesterol-rich diet significantly increased total serum cholesterol (c), and LDL (d) levels after angioplasty in rats. The data were expressed as means \pm SEM. $n=8$ per group. ${ }^{*} P<0.05$ versus normal diet group.

a fibroblast-like spindle-shaped morphology (Figure 1(b)). More than $95.69 \%$ of cells isolated from bone marrow were positive for CD90, and negative for the early hematopoietic marker CD34, indicating specific phenotype of BM-MSCs markers.

\subsection{The Profile of Lipid Level in Rats with Cholesterol-Rich} Diet. Baseline of serum lipid content before received cholesterol-rich diet did not significantly differ among three groups. The addition of cholesterol to the diet significantly increased total serum cholesterol, and LDL levels. Procedures of balloon angioplasty and BM-MSCs transplantation did not affect plasma lipid levels in this study (Figures 1 (c) and $1(\mathrm{~d}))$.

3.3. Effect of BM-MSCs on Vascular Remodeling after Balloon Angioplasty. To address vascular remodeling in vivo, histological analysis of vascular wall was assessed at 6 weeks after balloon angioplasty. Ruptured internal elastic lamina was present at the site of lesion, whereas splitting of the intima was replaced with significant neointimal formation after balloon angioplasty in injury group and MSC group. Data from histomorphometric parameters depicted that LA, IELA, EELA, and remodeling index significantly decreased after balloon angioplasty (LA: $1.632 \pm 0.043 \mathrm{~mm}^{2}$ versus $1.335 \pm$ $0.056 \mathrm{~mm}^{2}$ versus $1.133 \pm 0.074 \mathrm{~mm}^{2}$; IELA: $1.632 \mathrm{~mm}^{2} \pm$ $0.043 \mathrm{~mm}^{2}$ versus $1.414 \mathrm{~mm}^{2} \pm 0.073 \mathrm{~mm}^{2}$ versus $1.185 \mathrm{~mm}^{2} \pm 0.086 \mathrm{~mm}^{2}$; EELA: $2.403 \mathrm{~mm}^{2} \pm 0.067 \mathrm{~mm}^{2}$ versus $1.509 \pm 0.058 \mathrm{~mm}^{2}$ versus $1.308 \pm 0.044$; remodeling index: 1 versus $0.628 \pm 0.0293$ versus $0.544 \pm 0.0217$; control versus injury group versus MSC group, resp., $P<0.05$ ), whereas significant neointimal area and percentage of stenosis were increased, compared with control (neointimal area: 0 versus $0.078 \pm 0.015 \mathrm{~mm}^{2}$ versus $0.098 \pm 0.019 \mathrm{~mm}^{2}$, $P<0.05$; percentage of stenosis: 0 versus $18.20 \pm 1.09 \%$ versus $30.58 \pm 1.21 \%$; control versus injury group versus MSC group, resp., $P<0.05)$. BM-MSCs transplantation significantly aggravated vascular negative remodeling when compared with injury group, characterized by the decrease of LA, IELA, EELA, and remodeling index and increased neointimal area and percentage of stenosis (Figure 2). 

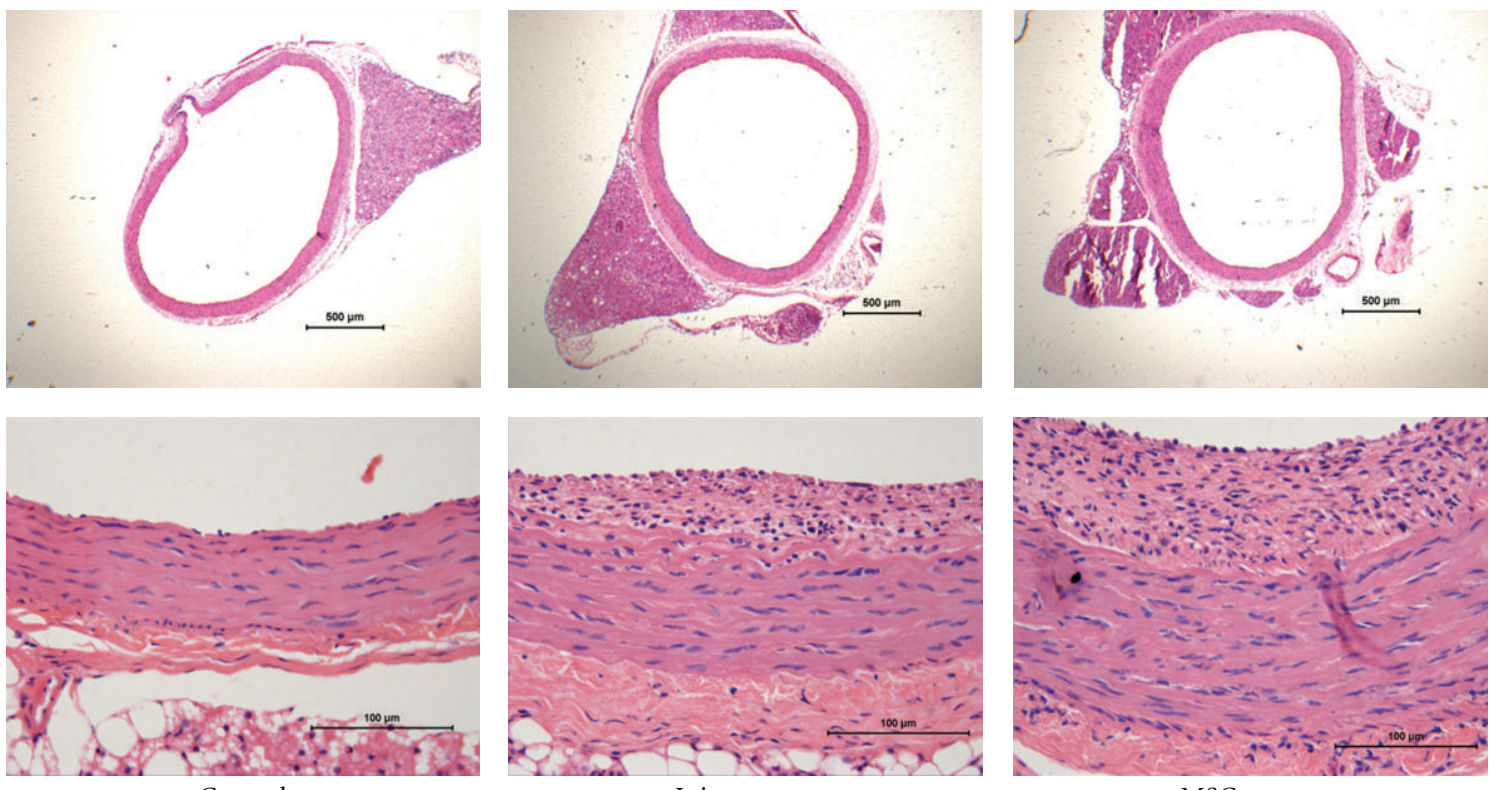

(a)
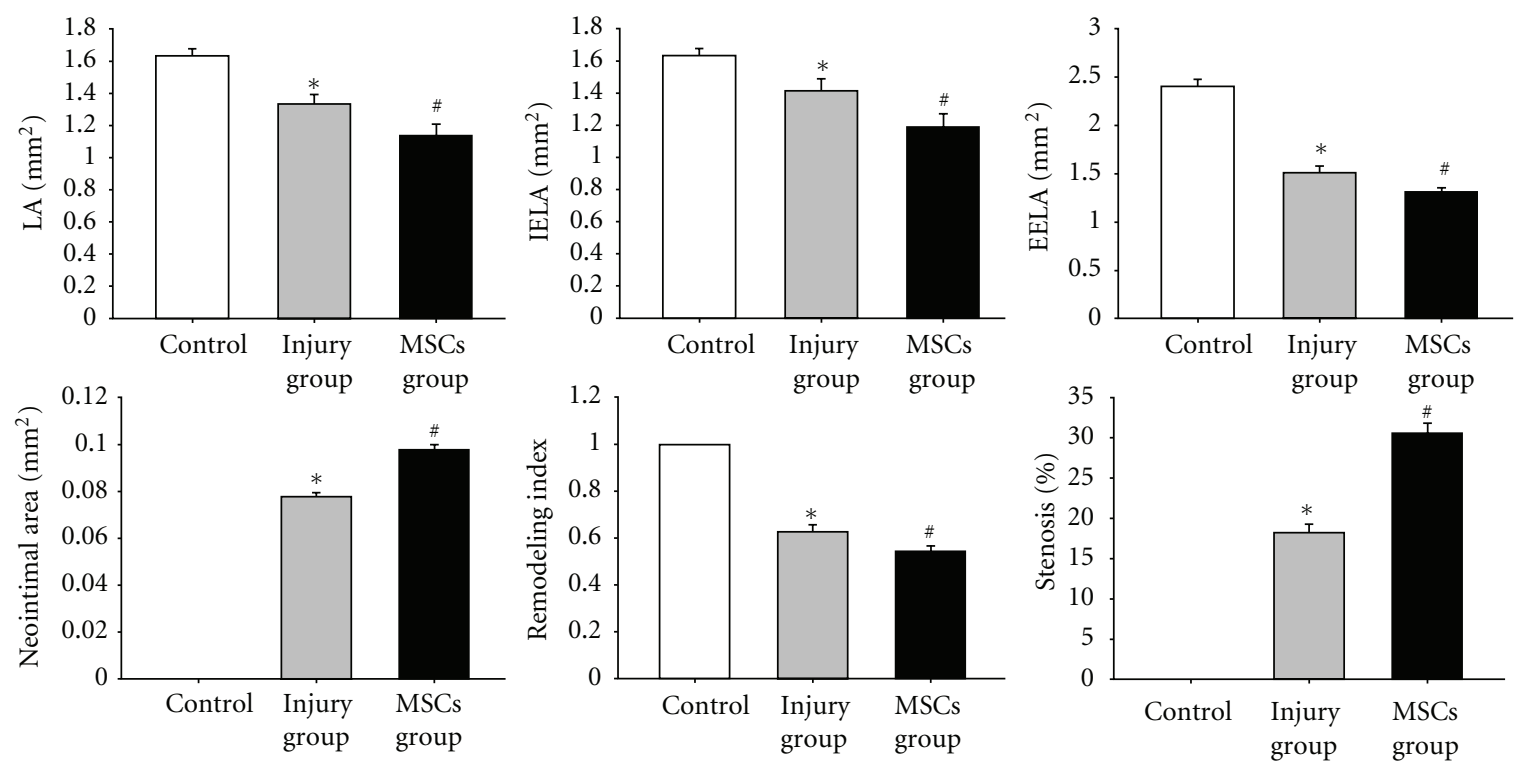

(b)

FIGURE 2: Effect of BM-MSCs on vascular remodeling after balloon angioplasty. Vascular tissues were stained with hematoxylin-eosin for morphometric analysis. (a) Ruptured internal elastic lamina was present at the site of lesion, whereas splitting of the intima was replaced with significant neointimal formation after balloon angioplasty. (b) Histomorphometric parameters including LA, IELA, EELA, remodeling index, neointimal area, and percentage of stenosis indicated vascular negative remodeling. The scale bar indicated $500 \mu \mathrm{m}$ (top row, 40x) and $100 \mu \mathrm{m}$ (bottom row, 400x), respectively. The data were expressed as means \pm SEM. $n=8$ per group. ${ }^{*} P<0.05$ versus control, ${ }^{\#} P<0.05$ versus injury group.

3.4. Effect of BM-MSCs on Neointimal Formation after Balloon Angioplasty. To investigate the neointimal formation in vivo, the PCNA index was evaluated. PCNA-stained nuclei were detected in the neointimal and medial layers of vascular wall at 6 weeks after vascular denudation (Figure 3). Data demonstrated that BM-MSCs resulted in a significant increase in the neointimal area $\left(0.078 \pm 0.015 \mathrm{~mm}^{2}\right.$ versus $\left.0.098 \pm 0.019 \mathrm{~mm}^{2}, P<0.05\right)$, PCNA index $(23.91 \pm 6.59 \%$ versus $43.11 \pm 5.31 \%, P<0.05$ ), and percentage of stenosis $(18.20 \pm 1.09 \%$ versus $30.58 \pm 1.21 \%, P<0.05)$ compared with injury group.

\subsection{Effect of BM-MSCs on Vascular Calcification after Balloon Angioplasty. To further characterize vascular calcification in}




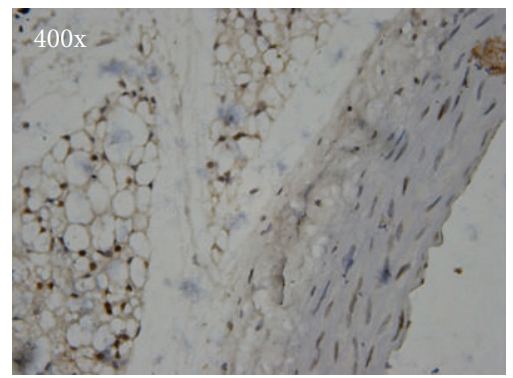

Control

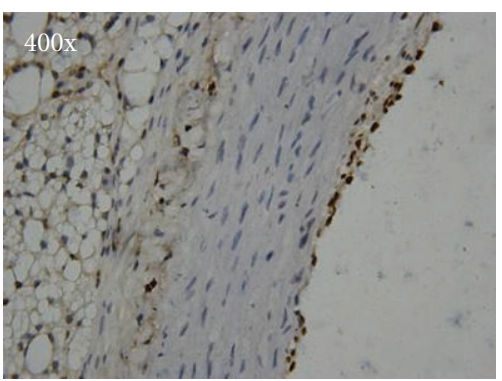

Injury group

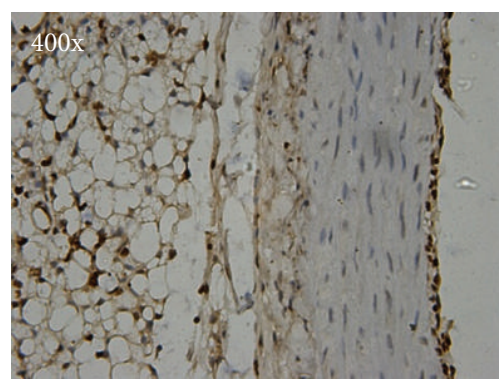

MSCs group

(a)

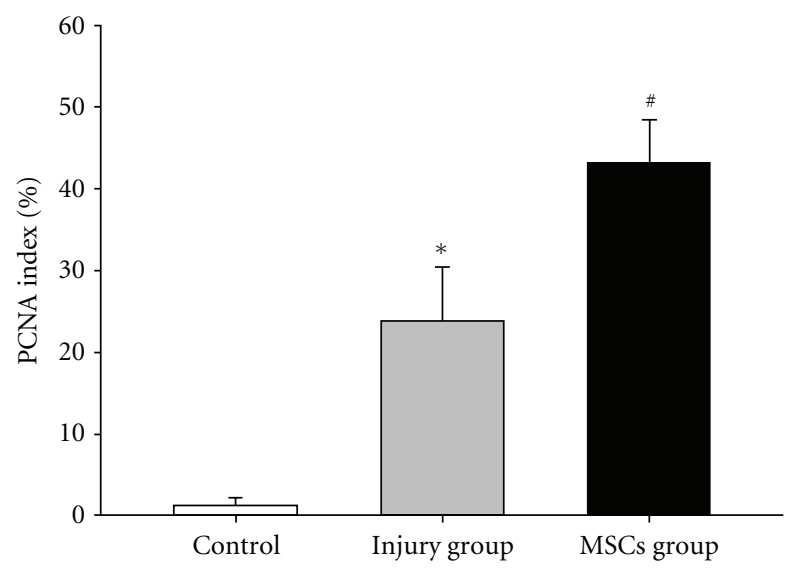

(b)

FIGURE 3: Effect of BM-MSCs on neointimal formation after balloon angioplasty. (a) The PCNA expression was evaluated immunohistochemically to investigate proliferative activity in vascular tissue. PCNA-stained nuclei were detected in the neointimal and medial layers of vascular wall at 6 weeks after vascular denudation. (b) PCNA index was significantly increased after BM-MSCs transplantation compared with injury group. The data were expressed as means \pm SEM. $n=8$ per group. ${ }^{*} P<0.05$ versus control, ${ }^{\#} P<0.05$ versus injury group.

lesions, von Kossa staining was performed. Typical of calcification, revealing black metallic deposit, was obviously visualized in medial layers in MSC group, compared control and injury group (Figure 4(a)). In view of association between bone morphogenetic protein-2 (BMP-2) and vascular calcification, the expression of BMP-2 was determined by Immunofluorescence staining. Results indicated that BMP-2 was strongly expressed in the neointimal and medial layers of vascular wall (Figure 4(b)). These results indicated that BMMSCs-induced vascular calcification might be associated with upregulation of BMP-2. To track the fate of BM-MSCs, BM-MSCs were prelabeled by DAPI. Labeled BM-MSCs were homing in the intimal layers at 6 weeks after angioplasty, indicating a role of BM-MSCs in the pathological changes of vascular remodeling and calcification (Figure 4(c)).

\section{Discussion}

The salient findings from our current study indicated that BM-MSCs transplantation triggered vascular remodeling and calcification after balloon angioplasty in hyperlipidemic rats. These results highlighted the potential importance of complication caused by BM-MSCs which should keep in mind in the therapeutic application.
Up to now, clinical trials for the treatment of ischemic heart diseases and heart failure using bone marrow cells were rapidly increased. Cell therapy has been regarded as a potential clinical strategy for cardiovascular diseases. However, intensive concerns about deleterious effects received significant attention, such as tumorigenicity, arrhythmia, and unregulated differentiation. Studies from Losordo reported for the first time that severe intramyocardial calcification was detected after transplantation of unselected BM cells in acute myocardial infarction [6]. The vascular remodeling and calcification were observed after balloon injury in hyperlipidemic rats. It is plausible that unselected BM-MSCs therapy could lead to unexpected complication in coronary heart disease patients with hyperlipidemia. Actually, patients with coronary heart diseases complicated by hyperlipidemia were clinically prevalent. Particularly, intracoronary delivery of BM-MSCs was performed after successful percutaneous coronary intervention in most of clinical trials. Thus, the gross adverse effect observed in the study could be clinically significant.

Increasing evidence indicated a link between bonemarrow-derived cells and the pathogenesis of atherosclerosis. Circulating myeloid calcifying cells were identified and found to contribute to ectopic vascular calcification in type 2 


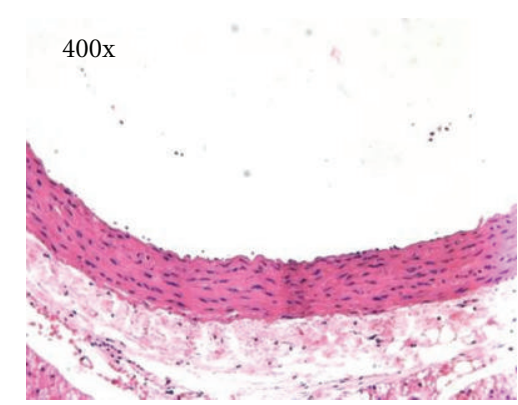

Control

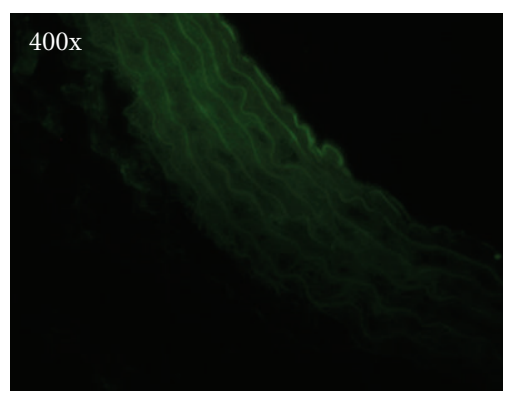

Control

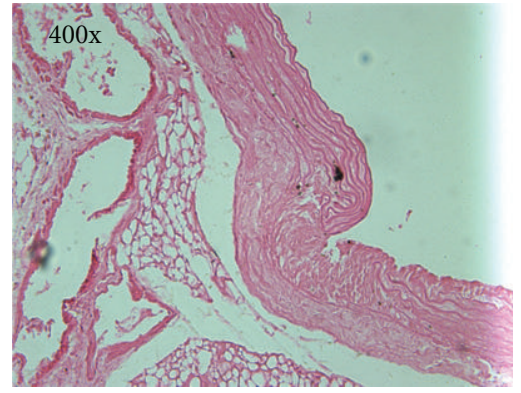

Injury group

(a)

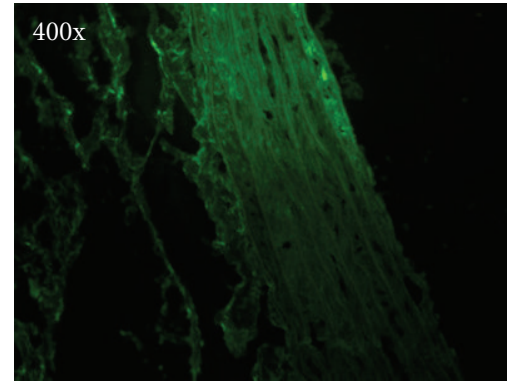

Injury group

(b)

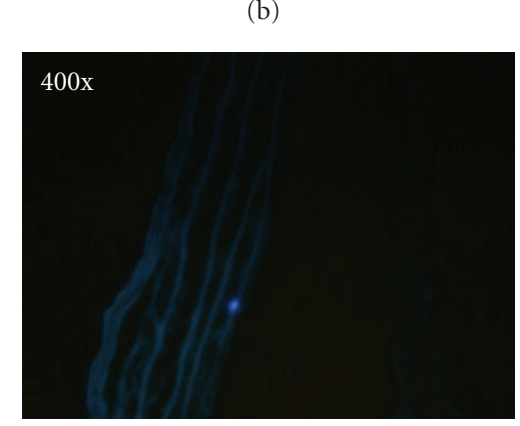

(c)

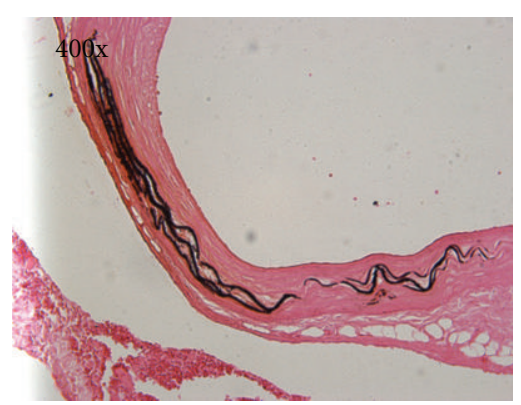

MSCs group

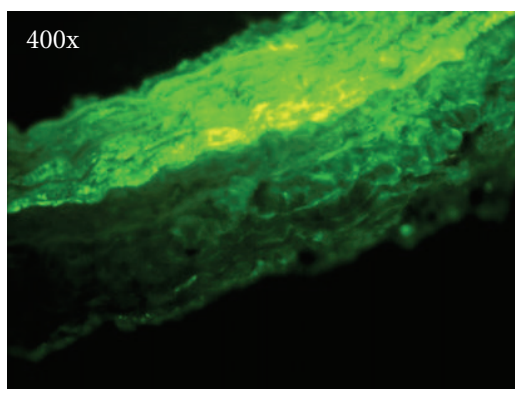

MSCs group

FIGURE 4: Effect of BM-MSCs on vascular calcification after balloon angioplasty and the fate of transferred BM-MSCs. (a) Von Kossa staining was performed to detect vascular calcification in lesions black metallic deposit was obviously visualized in medial layers in MSC group, compared control and injury group. (b) Immunofluorescence staining was performed to determine the expression of BMP-2. Results indicated that upregulated BMP-2 was strongly expressed in the neointimal and medial layers of vascular wall in MSC group, compared Control and Injury groups. (c) Labeled BM-MSCs were homing in the intimal layers at 6 weeks after angioplasty. $n=8$ per group.

diabetes [12]. Previous studies demonstrated that transferred BM-MSCs were homing to the vascular lesion and markedly increased atherosclerotic lesion size [7]. In this study, the fate of transferred BM-MSCs were traced and found to present at a low density in the vascular sections. However, the homing BM-MSCs may be underestimated, as quenching of the DAPI cannot be completely excluded at 6 weeks after balloon injury. It was indicated that BM-MSCs were potentially involved in atherosclerotic calcification.

The mechanisms by which transferred BM-MSCs modulate vascular calcification remain unclear. Compelling evidence suggested that vascular calcification was regulated by bone calcification regulatory factors locally expressed in blood vessels [13-15]. Paracrine BMP-2 caused the induction of osteoblast-like cells in intima and accelerated the atherosclerotic calcification in vivo [16]. BMP-2 was found to promote vascular calcification via increased phosphate uptake and induction of osteogenic phenotype modulation in smooth muscle cells [17]. Our observation that strongly expression of BMP-2 was detected in the neointimal and medial layers indicated a potential role of BMP-2 in the BMMSCs-induced calcification. Our data demonstrated that ox-LDL was synergistically promoted osteodifferentiation of BM-MSCs co-cultured with vascular smooth muscle cells in the presence of osteogenic inductor (unpublished data). It was suggested that BM-MSCs together with balloon injury and hyperlipidemia contribute to the formation of vascular calcification. 
Taken together, we demonstrated an important role of transferred BM-MSCs in vascular remodeling and calcification. These findings call for caution in potential adverse effects with respect to BM-MSCs transfer in the future clinical trials.

\section{Acknowledgments}

This study was supported by the Key Scientific Research Fund of Inner Mongolia Medical College (NY2004ZD007) and the Natural Scientific Research Fund of Inner Mongolia (2010MS1113).

\section{Authors' Contribution}

J. Liao and X. Chen contributed equally to this work.

\section{References}

[1] H. C. Quevedo, K. E. Hatzistergos, B. N. Oskouei et al., "Allogeneic mesenchymal stem cells restore cardiac function in chronic ischemic cardiomyopathy via trilineage differentiating capacity," Proceedings of the National Academy of Sciences of the United States of America, vol. 106, no. 33, pp. 14022-14027, 2009.

[2] E. A. Jones, S. E. Kinsey, A. English et al., "Isolation and characterization of bone marrow multipotential mesenchyma progenitor cells," Arthritis and Rheumatism, vol. 46, no. 12, pp. 3349-3360, 2002.

[3] A. R. Williams and J. M. Hare, "Mesenchymal stem cells: biology, pathophysiology, translational findings, and therapeutic implications for cardiac disease," Circulation Research, vol. 109, no. 8, pp. 923-940, 2011.

[4] J. O. Jeong, J. W. Han, J. M. Kim et al., "Malignant tumor formation after transplantation of short-term cultured bone marrow mesenchymal stem cells in experimental myocardial infarction and diabetic neuropathy," Circulation Research, vol. 108, no. 11, pp. 1340-1347, 2011.

[5] C. S. Kuo, K. Munakata, C. P. Reddy, and B. Surawicz, "Characteristics and possible mechanism of ventricular arrhythmia dependent on the dispersion of action potential durations," Circulation, vol. 67, no. 6 I, pp. 1356-1367, 1983.

[6] Y. S. Yoon, J. S. Park, T. Tkebuchava, C. Luedeman, and D. W. Losordo, "Unexpected severe calcification after transplantation of bone marrow cells in acute myocardial infarction.", Circulation, vol. 109, no. 25, pp. 3154-3157, 2004.

[7] J. George, A. Afek, A. Abashidze et al., "Transfer of endothelial progenitor and bone marrow cells influences atherosclerotic plaque size and composition in apolipoprotein E knockout mice," Arteriosclerosis, Thrombosis, and Vascular Biology, vol. 25, no. 12, pp. 2636-2641, 2005.

[8] A. Saiura, M. Sata, Y. Hirata, R. Nagai, and M. Makuuchi, "Circulating smooth muscle progenitor cells contribute to atherosclerosis," Nature Medicine, vol. 7, no. 4, pp. 382-383, 2001.

[9] M. Sata, A. Saiura, A. Kunisato et al., "Hematopoietic stem cells differentiate into vascular cells that participate in the pathogenesis of atherosclerosis," Nature Medicine, vol. 8, no. 4, pp. 403-409, 2002.

[10] L. Lin, H. Gong, J. Ge et al., "High density lipoprotein downregulates angiotensin II type 1 receptor and inhibits angiotensin II-induced cardiac hypertrophy," Biochemical and
Biophysical Research Communications, vol. 404, no. 1, pp. 2833, 2011.

[11] C. Brasselet, E. Durand, F. Addad et al., "Collagen and elastin cross-linking: a mechanism of constrictive remodeling after arterial injury," American Journal of Physiology, vol. 289, no. 5, pp. H2228-H2233, 2005.

[12] G. P. Fadini, M. Albiero, L. Menegazzo et al., "Widespread increase in myeloid calcifying cells contributes to ectopic vascular calcification in type 2 diabetes," Circulation Research, vol. 108, no. 9, pp. 1112-1121, 2011.

[13] K. A. Hruska, S. Mathew, and G. Saab, "Bone morphogenetic proteins in vascular calcification," Circulation Research, vol. 97, no. 2, pp. 105-114, 2005.

[14] R. C. Johnson, J. A. Leopold, and J. Loscalzo, "Vascular calcification: pathobiological mechanisms and clinical implications," Circulation Research, vol. 99, no. 10, pp. 1044-1059, 2006.

[15] M. Ketteler, G. Schlieper, and J. Floege, "Calcification and cardiovascular health: new insights into an old phenomenon," Hypertension, vol. 47, no. 6, pp. 1027-1034, 2006.

[16] Y. Nakagawa, K. Ikeda, Y. Akakabe et al., "Paracrine osteogenic signals via bone morphogenetic protein-2 accelerate the atherosclerotic intimal calcification in vivo," Arteriosclerosis, Thrombosis, and Vascular Biology, vol. 30, no. 10, pp. 19081915, 2010.

[17] X. Li, H. Y. Yang, and C. M. Giachelli, "BMP-2 promotes phosphate uptake, phenotypic modulation, and calcification of human vascular smooth muscle cells," Atherosclerosis, vol. 199, no. 2, pp. 271-277, 2008. 


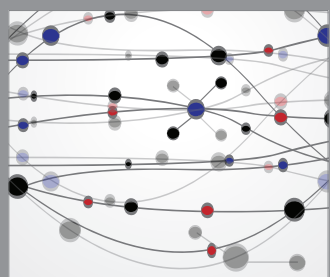

The Scientific World Journal
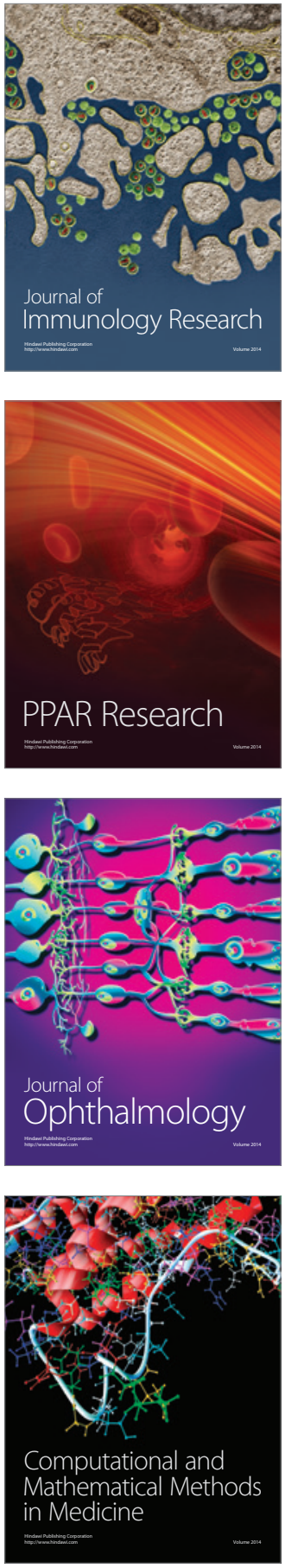

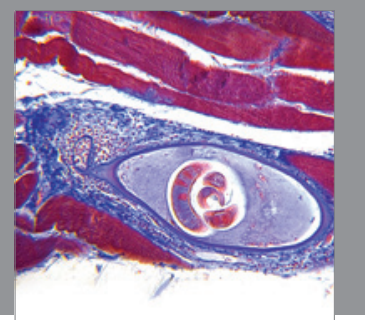

Gastroenterology

Research and Practice
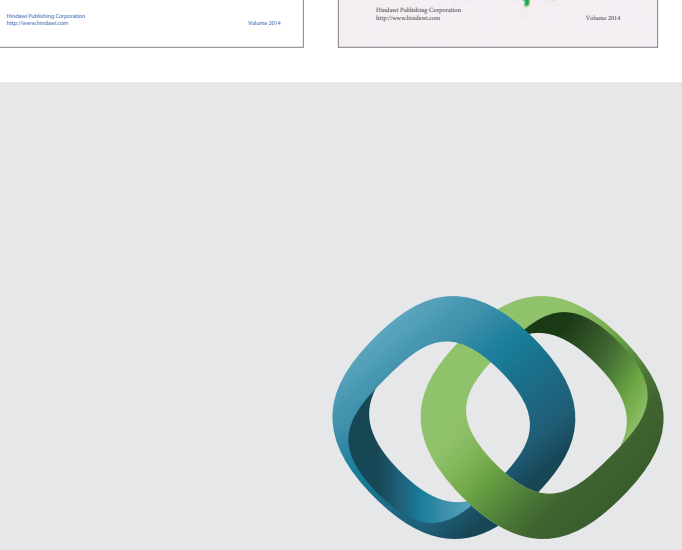

\section{Hindawi}

Submit your manuscripts at

http://www.hindawi.com
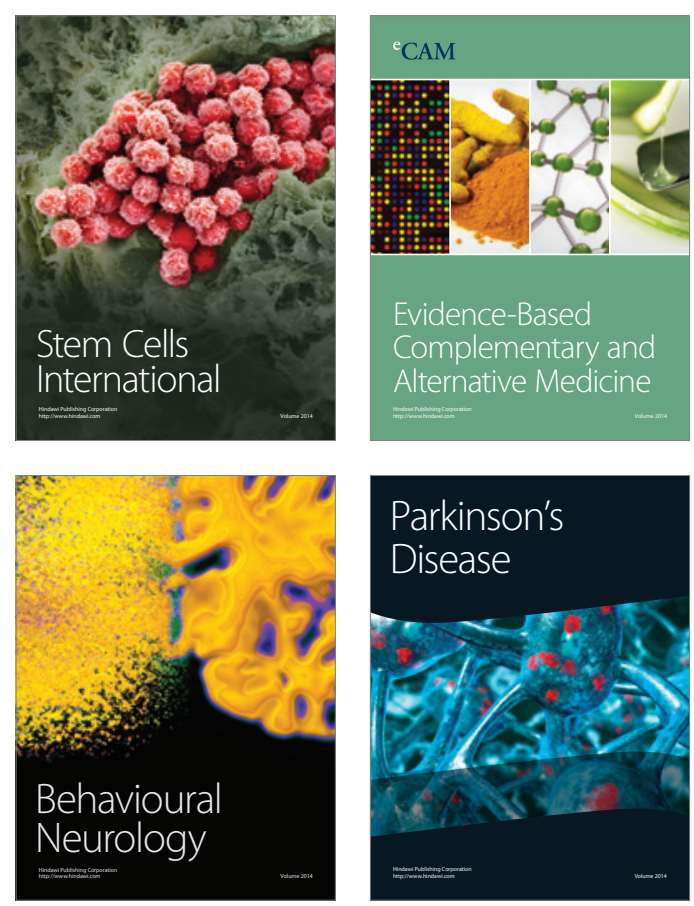

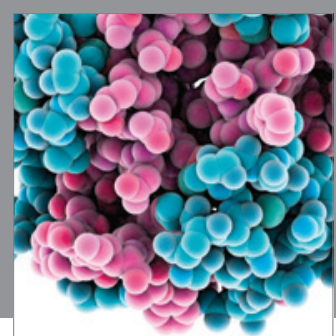

Journal of
Diabetes Research

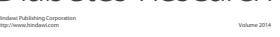

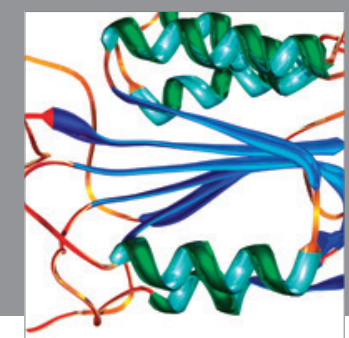

Disease Markers
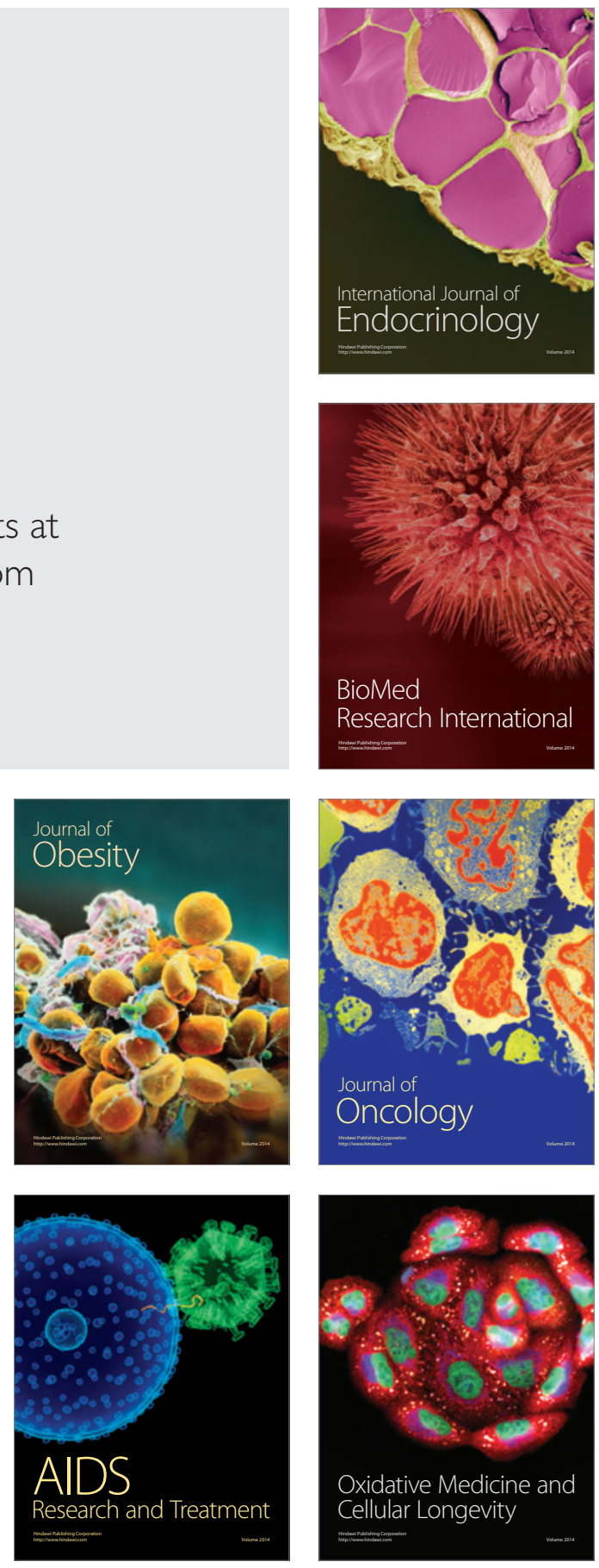